\title{
MOMENTUM-SPACE MONTE CARLO
}

\author{
P.A. AMUNDSEN ${ }^{1}$ \\ Nordita, Blegdamsvej 17, DK-2100 Copenhagen $\phi$, Denmark
}

\section{J. GREENSITE ${ }^{2}$}

Niels Bohr Institute, Blegdamsvej 17, DK-2100 Copenhagen $\phi$, Denmark

and

\section{T. STERLING}

Physics Department, University of Michigan, Ann Arbor, MI 48109, USA

Received 7 July 1984

Revised manuscript received 13 September 1984

\begin{abstract}
Using the quenched, reduced form of large- $N$ field theories, we show that it is possible to directly measure momentumspace Green functions, via Monte Carlo, without going through the intermediate step of measurement in position space plus Fourier transformation. This promises to be a useful tool for investigating the infrared structure of planar field theories. As an application (and test) of the method, we compute mass-gaps in the quenched $\mathrm{U}(N) \times \mathrm{U}(N)$ lattice chiral model, in $D=1$ and 2 dimensions.
\end{abstract}

In this letter we wish to point out an intriguing application of the quenched reduction method [1-3] to the numerical study of large- $N$ field theories. In standard Monte Carlo studies, it is customary to extract quantities of interest, such as mass-gaps and string tensions, from observables which fall off exponentially with distance, such as (position-space) Green functions and Wilson loops. Unfortunately, because of the exponential falloff, the separation of signal from noise in a Monte Carlo experiment often requires prohibitively large amounts of computer time, when distance scales greater than a few correlation lengths are involved. We will show here that this problem can be circumvented to some extent in the quenched, reduced formulation, by the direct calculation of momentumspace Green functions.

${ }^{1}$ Partly supported by NAVF. Address after July 1, 1984: Physik-Department T30, Technische Universität München, D-8046 Garching, West Germany.

2 Permanent address after September 1, 1984: Physics and Astronomy Department, San Francisco State University, 1600 Holloway Avenue, San Francisco, CA 94132, USA.
Consider, for example, extracting the mass-gap in the $\mathrm{U}(N) \times \mathrm{U}(N)$ lattice chiral model. The two-point function of this model, in the quenched reduced formulation, is given by

$$
\begin{aligned}
& G(x)=N^{-2}\left\langle\operatorname{Tr} U(x) U^{+}(0)\right\rangle=\frac{1}{N^{2}} \int_{-\pi}^{\pi}\left(\prod_{a=1}^{N} \frac{\mathrm{d}^{D} p_{a}}{(2 \pi)^{D}}\right) \\
& \times \sum_{i j} \exp \left[\mathrm{i}\left(p_{i}^{\mu}-p_{j}^{\mu}\right) x_{\mu}\right]\left\langle U_{i j} U_{j i}^{+}\right\rangle_{\text {red }},
\end{aligned}
$$

where

$$
\begin{aligned}
& \left\langle U_{i j} U_{j i}^{+}\right\rangle_{\mathrm{red}}=\frac{1}{Z} \int \mathrm{D} U U_{i j} U_{j i}^{+} \\
& \times \exp \left(2 \beta \sum_{\mu} \sum_{a b} \cos \left(p_{a}^{\mu}-p_{b}^{\mu}\right) U_{a b} U_{b a}^{+}\right)
\end{aligned}
$$

(no sum over $i, j$ ). Applying a simple Fourier transformation, this gives 


$$
\begin{aligned}
& G(p)=\sum_{x} G(x) \exp (-\mathrm{i} p \cdot x) \\
& =\frac{1}{N^{2}} \int_{-\pi}^{\pi} \prod_{a} \frac{\mathrm{d}^{D} p_{a}}{(2 \pi)^{D}} \sum_{i j}(2 \pi)^{D} \delta^{D}\left[p-\left(p_{i}-p_{j}\right)\right] \\
& \quad \times\left\langle U_{i j} U_{j i}^{+}\right\rangle_{\text {red }} .
\end{aligned}
$$

Alternatively, in the $N \rightarrow \infty$ limit, we can replace the integration over the $\left\{p_{a}^{\mu}\right\}$ by the choice of a single, uniformly distributed set of $N$ "master momenta" $\left\{p_{i}^{\mu *}\right\}$ (see refs. [3,4] for details), in which case

$G\left(p_{i}^{*}-p_{j}^{*}\right)=\left\langle U_{i j} U_{j i}^{+}\right\rangle_{\text {red }}^{*}$,

where \langle\rangle$_{\text {red }}^{*}$ means to evaluate the reduced matrix elements in (2) using a particular set of master momenta $\left\{p_{i}^{* \mu}\right\}$.

Now of course the long-distance behavior of $G(x)$, $x \rightarrow \infty$ is controlled by the infrared behavior of $G(p)$, $p \rightarrow 0$. And from (3) or (4) we see that $G(p), p \rightarrow 0$ is obtained from the expectation value of the quantity $\left\langle U_{i j} U_{j i}^{+}\right\rangle_{\mathrm{red}},\left(p_{i}-p_{j}\right) \rightarrow 0$. The crucial point is that, unlike $G(x)=\left\langle\operatorname{Tr} U(x) U^{+}(0)\right\rangle / N^{2}$, which falls off exponentially as $x \rightarrow \infty$, the expectation value $\left\langle U_{i j} U_{j i}^{+}\right\rangle_{\text {red }}$ approaches a constant as $\left(p_{i}-p_{j}\right) \rightarrow 0$, for any nonzero mass gap. This makes $G(p), p \rightarrow 0$ much easier to measure, in principle, than $G(x)$ at large $x$. In particular, if we make a simple one-pole approximation for the lattice propagator

$G^{-1}(p) \approx($ const. $) \times\left(\tilde{p}^{2}+\mu^{2}\right)$,

$\tilde{p}^{2} \equiv 2 \sum_{\mu=1}^{D}\left(1-\cos p_{\mu}\right)$,

then

$\mu^{2}=G^{-1}(0)\left(\mathrm{d} G^{-1} /\left.\mathrm{d} \tilde{p}^{2}\right|_{\tilde{p}=0}\right)^{-1}$,

so that we need only the value and slope of $G^{-1}(p)$ near $\widetilde{p}^{2}=0$ to determine the mass-gap. The validity of the one-pole approximation (5) is checked if $G^{-1}(p)$ is well approximated by a straight line.

In practice, we divide $p$-space into a large number $M(\approx 50)$ of subvolumes $V_{m}(m=1, \ldots, M)$, and measure the average value of $G(p)$ in each subvolume, i.e.

$G_{m}=\frac{1}{V_{m}} \int \mathrm{d}^{D} p \Delta_{m}(p) G(p)$
$\Delta_{m}(p)=1, p$ in subvolume $m$,

$=0$, otherwise,

( 7 cont'd)

is the average of $G(p)$ in subvolume $m$. Then from eq. (3) we have

$$
\begin{aligned}
G_{m} & =\frac{1}{N^{2}} \int \prod_{a} \frac{\mathrm{d}^{D} p_{a}}{(2 \pi)^{D}} \sum_{i j} \frac{(2 \pi)^{D}}{V_{m}} \Delta_{m}\left[\left(p_{i}-p_{j}\right)\right] \\
& \times\left\langle U_{i j} U_{j i}^{+}\right\rangle_{\text {red }},
\end{aligned}
$$

which is to be calculated by the Monte Carlo method.

The procedure for evaluating ( 8 ) is by a Monte Carlo-within-a-Monte Carlo, i.e. a sequence of Monte Carlo evaluations of eq. (2), with each evaluation using a different set of quenched momenta $\left\{p_{a}\right\}, a=1, \ldots, N$. Each set of quenched momenta is generated stochastically with uniform weighting in the $D$-dimensional $p$ space volume $-\pi \leqslant p^{\mu} \leqslant \pi, \mu=1, \ldots, D$. Denote the $k$ th set of quenched momenta by $\left\{p_{a}^{(k)}\right\}, k=1, \ldots, K$, and let $\left\langle U_{i j} U_{j i}^{+}\right\rangle^{k}$ denote the expectation value of $\left\langle U_{i j} U_{j i}^{+}\right\rangle$in the $k$ th quenched momenta set. Then

$$
\begin{aligned}
G_{m} & =\frac{1}{K} \sum_{k=1}^{K} \frac{1}{n(m, k)} \sum_{i \neq j}\left\langle U_{i j} U_{j i}^{+}\right\rangle^{(k)} \\
& \times \Delta_{m}\left(p_{i}^{(k)}-p_{j}^{(k)}\right)
\end{aligned}
$$

is the value of $G_{m}$ determined from the sequence of Monte Carlo's, where

$n(m, k)=\sum_{i \neq j} \Delta_{m}\left(p_{i}^{(k)}-p_{j}^{(k)}\right)$

is the number of relative momenta $p_{i}^{(k)}-p_{j}^{(k)}$ in the $k$ th momenta set which lie in the $m$ th subvolume of $p$-space.

Let $\tilde{p}^{2}(m)$ denote the average value of $\tilde{p}^{2}$ in the subvolume $m$. The last step in finding the mass-gap $\mu$ is to plot $G_{m}^{-1}$ as a function of $\widetilde{p}^{2}(m)$, and extract $\mu^{2}$ from eq. (6).

For the $D=1$ dimensional chiral model, we have defined the $m$ th subvolume to be the range $(m=1, \ldots$, 50)

$\frac{1}{50}(m-1) \pi<|p| \leqslant \frac{1}{50} m \pi$,

with

$\tilde{p}^{2}(m)=2\left\{1-\cos \left[\frac{1}{50}(m-1 / 2) \pi\right]\right\}$.

Following the procedure outlined above, we have com- 


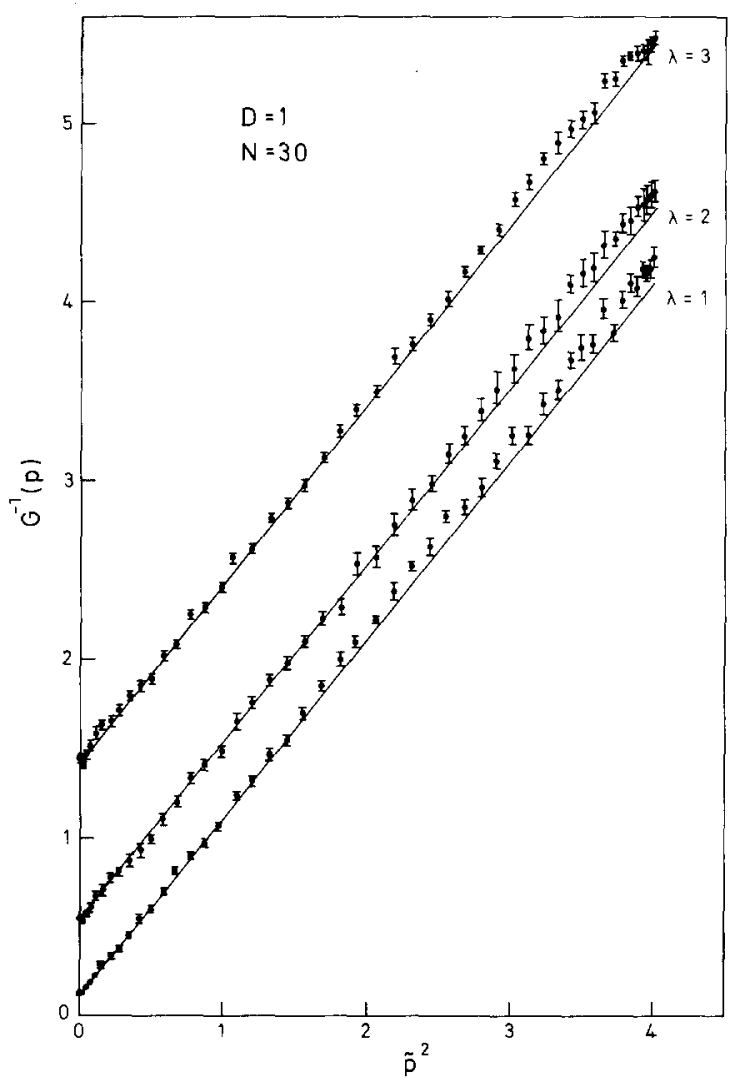

Fig. 1. The inverse two-point function $G^{-1}(p)$ versus $\tilde{p}^{2}$ at $N=30, D=1$ dimension, for couplings $\lambda=1,2$ and 3. Each data point represents the average of $G^{-1}(p)$ in a small subinterval $V_{m}$. For points without error bars, the error is less than the size of the solid dot.

puted $G(p)$ and $\mu^{2}$ throughout the range of couplings $\lambda=N / \beta$. In fig. 1 we show our results for $G^{-1}(p)$ as a function of $\tilde{p}^{2}$ (or, strictly speaking, $G_{m}^{-1}$ versus $\tilde{p}^{2}(m)$ ) for coupling values $\lambda=1,2,3$ and $N=30$. The coupling $\lambda=1$ is in the weak-coupling region, $\lambda=3$ is at strongcoupling, and $\lambda=2$ is the third order Gross-Witten transition point [5]. In all three cases the data is fit quite well by a straight line, and $\mu^{2}$ can be extracted from (6). The straight line is a least-squares fit for the values $1 \leqslant m \leqslant 20$ [note that the diagonal $(i=j)$ contributions at $\tilde{p}=0$ are excluded]. The error bars are calculated by assuming the contributions for different sets $\left\{p_{a}^{(k)}\right\}$ to be statistically independent (which was verified from standard $\chi^{2}$ analysis). In general, we found 30 sets of momenta for $N=10$ and 10 sets for $N=20$ and 30 to be sufficient. For each set of mo- menta we found that 100 updates of the full matrix was enough to remove dependence on the initial conditions, with data taken on the subsequent 300 sweeps. The matrices were updated following Okawa's [6] procedure.

Since the leading corrections to the $N=\infty$ result for $\mu$ at finite $N$ are expected to be of order $1 / N$ (in the quenched model), we have fit our data for $N=10$, 20,30 to the assumed form

$\mu=\mu_{\infty}+c / N$.

In fig. 2 we show typical extrapolations for $\lambda=2$ in $D=1$ dimensions, and $\lambda=2.5$ in $D=2$ dimensions (see below). The data is fit quite well by a straight line, so the extrapolation to $N=\infty$ seems reliable. In fig. 3 we plot the extrapolated mass-gap $\mu_{\infty}$ versus $\beta / N=\lambda^{-1}$, compared against the exact Gross-Witten result. The agreement is clearly very good. Similar excellent agreement was found for the extrapolated expectation values of the action.

As the computer time available to us was rather limited, we were unable to carry out a thorough calculation of $\mu$ versus $\lambda^{-1}$ in $D=2$ dimensions, to check for the proper scaling according to asymptotic freedom. We have, however, computed the mass-gap at a few selected values of $\lambda$ to check the practicality of our method in $D=2$ dimensions. In the two-dimensional case, we have defined the $m$ th subvolume as the

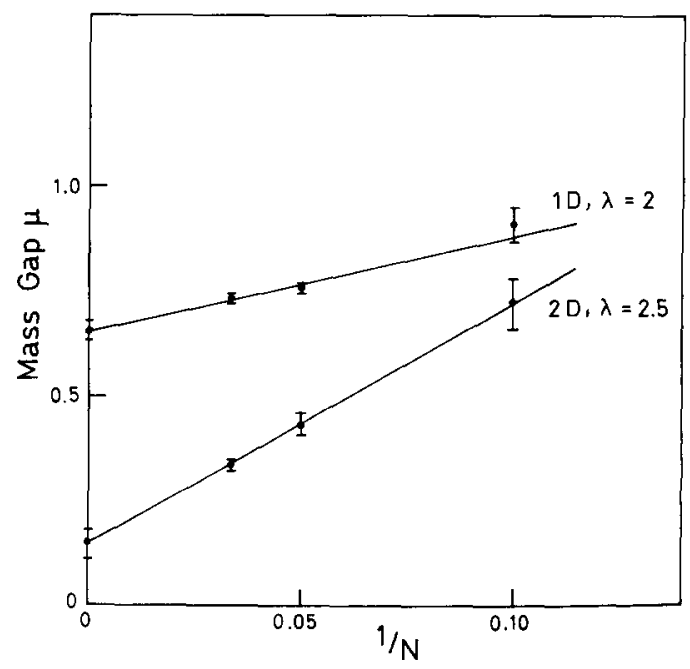

Fig. 2. Extrapolation of the mass-gap $\mu$ to $N=\infty$, from data at $N=10,20$ and 30. Data shown is for $\lambda=2$ in $D=1$ dimension, and $\lambda=2.5$ in $D=2$ dimensions. 


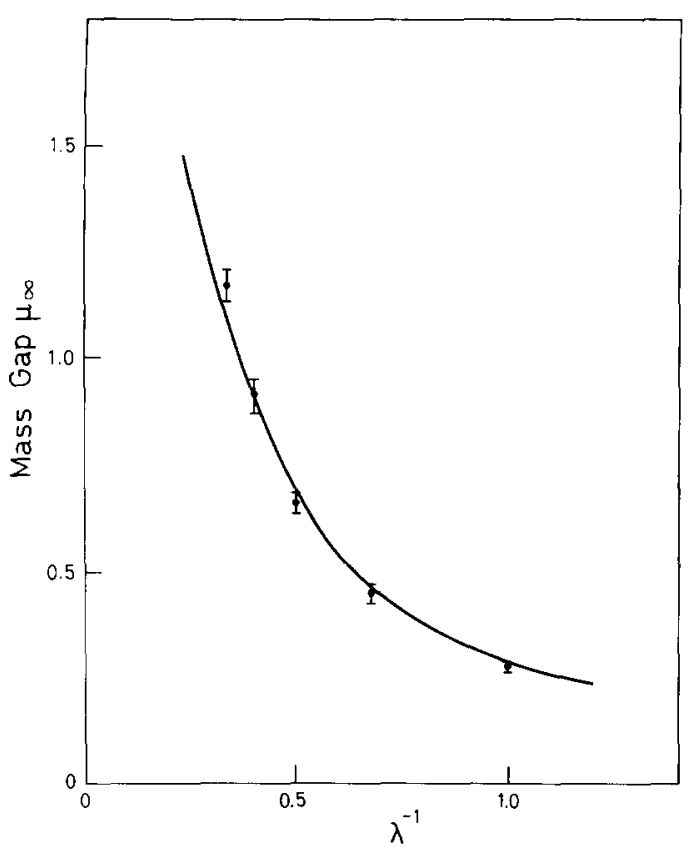

Fig. 3. Mass-gap $\mu_{\infty}$ as a function of $\lambda^{-1}$ in $D=1$ dimension. Solid dots are the (extrapolated) data points; the curve is the exact Gross-Witten result.

subspace in which

$\frac{8}{50}(m-1)<\widetilde{p}^{2} \leqslant \frac{8}{50} m \quad(1 \leqslant m \leqslant 50)$.

The reason for the different choice of $V_{m}$, compared to (11), is to avoid that $n(m, k)$ becomes too strongly dependent on $m$.

In fig. 4 we plot $G^{-1}(p)$ versus $\tilde{p}^{2}$ at $\lambda=4.44$ and $\lambda=2.5$, in the strong- and weak-crossover coupling regions, respectively, at $N=30$. Once again, the data for $G^{-1}\left(\widetilde{p}^{2}\right)$ is fit very well by a straight line. The massgaps at $N=\infty$, extrapolated from data at $N=10,20$ and 30 (see fig. 2) were determined to be

$\mu_{\infty}=1.19 \pm 0.06, \lambda=4.44$,

$\mu_{\infty}=0.15 \pm 0.04, \lambda=2.5$,

in units of inverse lattice spacing ${ }^{\ddagger 1}$. The data point at $\lambda=2.5$ required $1 \frac{1}{2} \mathrm{~h} C P$ time on the CYBER 172 .

Das and Kogut [7] have previously computed the position-space two-point function $G(x)$ in the $t$ wisted

${ }^{\ddagger 1}$ At very strong coupling $(\lambda=10)$ the statistics did not allow for meaningful ex trapolation to $N=\infty$.

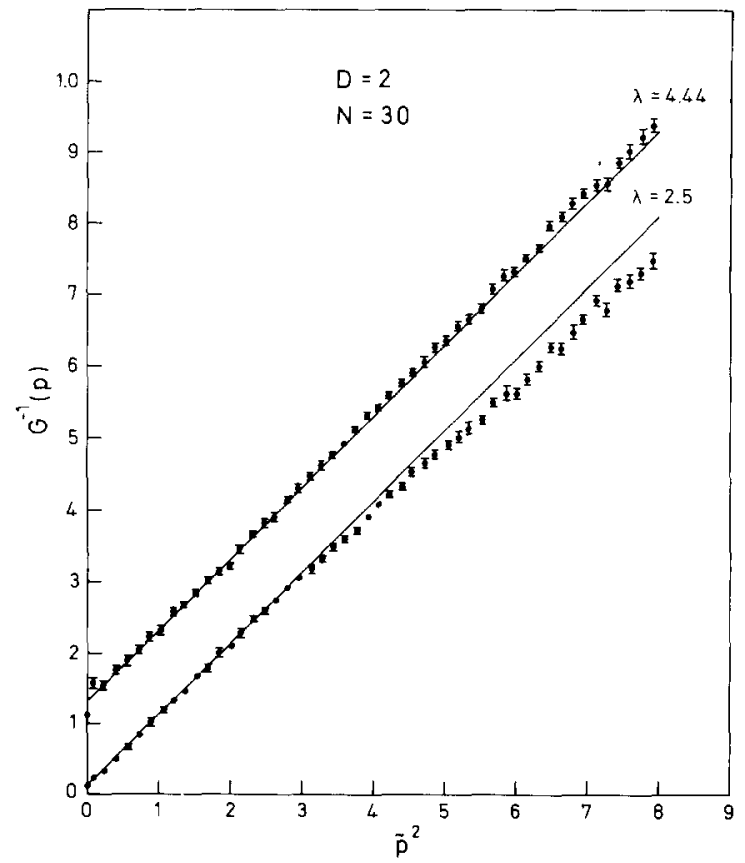

Fig. 4. $G^{-1}(p)$ versus $\widetilde{p}^{2}$ at $N=30$ in $D=2$ dimensions, at $\lambda=4.44$ (strong coupling), and $\lambda=2.5$ (weak coupling).

reduced $\mathrm{U}(N) \times \mathrm{U}(N)$ chiral model, via Monte Carlo, and found surprising non-analytic behavior $[G(x)<0$ for some $x$ ] near the crossover. Klinkhamer [8] has recently suggested an explanation for the apparent non-analyticity in the Monte Carlo data, based on nontrivial extrema of the twisted, reduced action. In the quenched, reduced model used in this paper there was no sign of non-analyticity, at least not at the couplings where we have measured. In fact, $G(p)$ seems to be fit remarkably well in $D=1$ and 2 dimensions by the onepole ansatz (5).

We believe that the method outlined above can provide an accurate determination, not only of masses, but also effective couplings defined from appropriate three- and four-point momentum-space Green functions, for a variety of large- $N$ matrix models. For $Q C D$, in particular, it would be especially interesting to measure the product of Bars' corner variables [9]

$$
\begin{gathered}
W\left[\left(p_{i}^{*}-p_{k}^{*}\right)^{x},\left(p_{l}^{*}-p_{j}^{*}\right)^{y}\right] \\
=\left\langle B_{x y}^{i j} B_{y x}^{j k} B_{x y}^{k l} B_{y x}^{l i}\right\rangle_{\mathrm{red}}^{*},
\end{gathered}
$$

which can be recognized as the momentum-space ver- 
sion of rectangular $L \times W$ Wilson loops via the relation

$$
\begin{gathered}
W\left[C_{L W}\right]=\int \prod_{a} \frac{\mathrm{d}^{D} p}{(2 \pi)^{D}}{ }_{i j k l} \exp \left[\mathrm{i}\left(p_{i}-p_{k}\right)^{x_{\mathrm{L}}}\right] \\
\quad \times \exp \left[\mathrm{i}\left(p_{l}-p_{j}\right)^{y \mathrm{~W}}\right]\left\langle B_{x y}^{i j} B_{y x}^{j k} B_{x y}^{k l} B_{y x}^{l i}\right\rangle_{\mathrm{red}} .
\end{gathered}
$$

In practice one would evaluate $W\left(p_{x}, p_{y}\right)$ by averaging over many sets of quenched momenta, as in the treatment of the chiral model above. The behavior of $W\left(p^{x}, p^{y}\right)$ at $p \rightarrow 0$ may shed some light on the proper expression, in momentum space, of the QCD confinement criterion.

One drawback to the momentum-space method which should be mentioned is the restriction to the quenched reduced formulation, which is cumbersome to apply numerically, and which has much larger finitesize (finite- $N$ ) effects than the twisted reduced formulation. It was seen in fig. 2 that substantial $1 / N$ deviations in the 2D chiral model can be isolated and eliminated by a straightforward extrapolation procedure. The feasibility of this sort of procedure, in terms of computer time, for $\mathrm{SU}(\infty)$ gauge theory in $D=4$ dimensions remains to be seen.

Another point is that the extraction of a mass-gap requires some assumptions about the form of the twopoint function, since the $p \rightarrow 0$ limit does not automatically single out the lowest mass excitation. In this paper we have fit $G^{-1}(p)$ to a straight line, i.e. the onepole ansatz (5). The justification for this ansatz is simply that the data itself is so obviously linear, not just near $p=0$ but over at least half the momentum range for the $D=1$ and 2 dimensional chiral models (see fig. 4). Now the general form of a two-point function, involving bound states, thresholds, etc., is rather complicated, so the linearity for the $D=2$ model is somewhat surprising. The success of the one-pole ansatz may be at least partially explained by the recent result of Wiegmann [10], showing that multiparticle bound states disappear at $N=\infty$ in the $2 \mathrm{D} \operatorname{SU}(N) \times \operatorname{SU}(N)$ chiral model. In other theories, of course, the higher excitations would contribute, and a more complicated ansatz for $G^{-1}(p)$ may be required. It should be noted that "contamination" of the two-point function with higher excitations is also a problem in standard Monte Carlo simulations. In principle this contamination can be eliminated, in standard simulations, by measurements at sufficiently large distance scales; but then the signal from the lowest excitation may also sink into the noise. In future studies with the momentum-space method, it would be interesting to try a best-fit of $G^{-1}(p)$ to a multiple-pole ansatz

$G^{-1}(p)=\left(\sum_{i} \frac{A_{i}}{p^{2}+\mu_{i}^{2}}\right)^{-1}$,

to allow for (and find the mass of) the higher excitations.

The computations on the RBK CYBER 172 were made possible through the courtesy of the Department of Physics at the University of Oslo, and we are very grateful to Kjell Aashamar for arranging this.

\section{References}

[1] G. Bhanot, U. Heller and H. Neuberger, Phys. Lett. 113B (1982) 47.

[2] G. Parisi and Y.C. Zhang, Phys. Lett. 114B (1982) 319.

[3] D. Gross and Y. Kitazawa, Nucl. Phys. B206 (1982) 440.

[4] J. Greensite and M. Halpern, Nucl. Phys. B211 (1983) 343.

[5] D. Gross and E. Witten, Phys. Rev. D21 (1980) 446.

[6] M. Okawa, Phys. Rev. Lett. 49 (1982) 353.

[7] S. Das and J. Kogut, Nucl. Phys. B235 [FS11] (1984) 521.

[8] F. Klinkhamer, Nucl. Phys. B240 [FS12] (1984) 270.

[9] I. Bars, Phys. Lett. 116B (1982) 57.

[10] P. Wiegmann, Phys. Lett. 141B (1984) 217. 